\title{
Primary brainstem death: a clinico-pathological study
}

\author{
J OGATA, * M IMAKITA, $\dagger$ C YUTANI, $\dagger$ S MIYAMOTO, $\ddagger$ H KIKUCHI \\ From the Research Institute, ${ }^{*}$ Department of Pathology, $†$ Department of Neurosurgery, $\ddagger$ National \\ Cardiovascular Centre, Osaka, Japan
}

SUMMARY A case of primary brainstem death in a man with surgically treated cerebellar haemorrhage is reported. Necropsy revealed extensive necrosis confined to the brainstem and cere- $\frac{\omega}{\sigma}$ bellum. The absence of diabetes insipidus and the persistence of electroencephalographic activity $\stackrel{\mathbb{D}}{\Omega}$ were the characteristic clinical features of the case. This differentiates the condition from so-called $ڤ$ "whole brain death". Analysis of three further cases with acute vascular lesions of the brainstem or $\vec{\circ}$ cerebellum, shown at necropsy, revealed that primary brainstem death with prolonged somatic survival can occur in specific circumstances after surgical intervension

Brainstem death, as encountered in clinical practice, is nearly always a secondary phenomenon. It is the infratentorial repercussion of supratentorial events and has been described as the "physiological core" of "whole brain death" and as the main determinant of its clinical features and cardiac prognosis. ${ }^{1}$ Lesions to the upper and lower brainstem which deprive it of its function may however produce the neurological entity of primary brainstem death. ${ }^{2}$ Judicial hanging (which ruptures the brainstem between pons and medulla without causing primary injury to the cerebral hemispheres) is the commonest cause of such a situation. There have been very few reports of "brain death" with prolonged somatic survival caused by lesions confined to the brainstem. ${ }^{34}$ We here report a necropsied case of cerebellar haemorrhage reproducing the clinical features of brainstem death and with somatic survival lasting 27 days. The pathological examination revealed necrosis confined to the brainstem and cerebellum.

To determine the clinico-pathological characteristics of primary brainstem death in patients with infratentorial vascular lesions, necropsied cases with acute vascular lesions of the brainstem or cerebellum were then analysed.

Address for reprint requests: Jun Ogata, M.D., National Cardiovascular Centre Research Institute, 5-7-1 Fujishirodai, Suita, Osaka 565, Japan.

Present address (S.M., H.K.): Department of Neurosurgery, Kyoto University Medical School, Syogoin-Kawaramachi 54, Sakyo-ku, Kyoto 606, Japan

Received 4 September 1987 and in revised form 25 November 1987. Accepted 1 December 1987

\section{Case report}

A 47 year old hypertensive man was found lying on the floor In 10 minutes, he became unresponsive. The blood pressure $\vec{\sigma}$

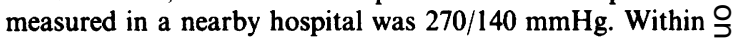
an hour, he was intubated because spontaneous respirati@ ceased. The blood pressure fell at one stage to $80 / 52 \mathrm{mmH}$. $>$ Three hours after the onset, he was transferred to the National Cardiovascular Center.

On admission, he was deeply comatose. Pupillary lighi $\vec{\emptyset}$ reactions were absent. The blood pressure was 146/196 $\mathrm{mmHg}$. The extremities were flaccid. Routine laboratory examination was normal. Computed tomography (CT) of the brain revealed a massive haematoma (measuring $6 \mathrm{~cm} \times$ $4.5 \mathrm{~cm}$ in its maximum diameters) involving the left cerebellar hemisphere and vermis with ventricular rupture. $\frac{\mathrm{Q}}{\mathrm{Q}}$ Dopamine and corticosteroid were administered for cardio- $\varrho$ vascular support. An emergency suboccipital craniotomy $\overrightarrow{\vec{\sigma}}$ was performed 4 hours after the onset; the dura over the 3 cerebellar hemispheres was so tense that the cerebellar tissue $\underset{\tau}{ }$ spouted out from the dural incision. Evacuation of the haematoma and extensive decompressive craniectomy were performed with opening of the foramen magnum and laminectomy of the first cervical spine. A fifth of the cere-? bellar tissue was also removed to provide an internal decom- $\frac{O}{3}$ pression. After the operation, the patient remained deeply comatose, and without spontaneous respiration. Because $\delta$ CT had shown dilated lateral ventricles, ventricular drainage was performed on the following day. The neurological status $\mathrm{O}$ remained unchanged until cardiac arrest occurred, in a state of progressive emaciation, 26 days after the onset of the
stroke.

Neurological evaluation during this period established the $N$ persistent presence of a clinical syndrome suggesting a dead brainstem. The patient had not been under the influence of $\mathrm{N}$ central nervous system depressant or neuromuscular block- $\mathrm{N}$ ing drugs. He was not hypothermic. He was comatose and $\bar{\sigma}$ apnoeic, although formal disconnection tests, with estimation of blood gases, were not performed. Ventilation was 


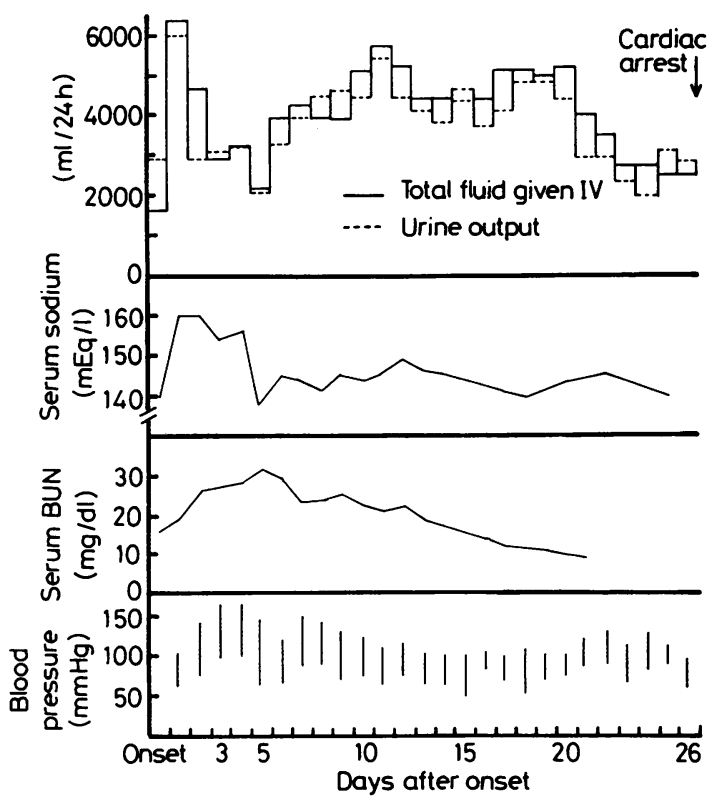

Fig 1 Clinical course after the stroke. (Blood pressure was measured at noon.)

ensured by a respirator (Servo Ventilator $900 \mathrm{C}$, SiemensElema, Sweden). The pupillary light reactions and corneal, oculocephalic and cough reflexes were absent. All four extremities were flaccid, and there was no spontaneous movement of the face or limbs. After the operation, the patient was given nothing by mouth, and received continuous intravenous fluid replacement with dehydrating agents such as mannitol and glycerol. The blood pressure was maintained without pharmacological support. Fluid balance was maintained. Though the serum values of sodium and blood urea nitrogen were elevated with massive urine output in the acute period, these values were normalised thereafter (fig 1). The serum creatinine which had been $1.4 \mathrm{mg} / \mathrm{dl}$ on admission, and $2.8 \mathrm{mg} / \mathrm{dl}$ on day 2 , returned to normal values within a few days.

Sensory evoked potentials on median nerve stimulation were absent on day 4. A monopolar electroencephalogram (EEG) obtained on day 7 showed disorganised irregular 5-6 $\mathrm{Hz}, 10-30 \mu \mathrm{V}$ theta activity. No response was noted to voice or painful stimuli. The amplitude and frequency of the activity attenuated to some degree on days 19 and 24. CT of the brain showed slight dilatation of the lateral ventricles on days 10 and 18 . Necropsy restricted to the brain was performed within an hour of cardiac arrest.

\section{Pathological findings}

Gross The cerebrum was not macerated and superficially showed no specific pathological changes. The brainstem and a large portion of the cerebellum were necrotic. The arteries at the base of the brain were slightly to moderately sclerotic but their lumen was patent. The superior sagittal and lateral sinuses were also patent. Upon sectioning, the cerebral hemispheres showed only slight dilatation of the lateral and third ventricles (fig 2). Most of the brainstem showed irregular and diffuse necrosis from the medulla oblongata to the midbrain (fig 3). The midbrain, other than the tegmentum, was preserved.

Microscopic The tegmentum of the midbrain, tegmentum and basis pontis, and medulla oblongata were almost completely devoid of neurons and replaced by a mass of fatty macrophages and proliferating astrocytes in keeping with a process of 27 days duration (figs 4, 5). Most of the cerebellar tissue was also necrotic. The neurons were lost or showed ischaemic change with proliferating astrocytes in the Sommer's sectors of the both hippocampi and the subiculi. Sections from the cerebrum showed no specific pathological changes of the cortical and subcortical gray matter. There was some rarefaction of the deep white matter. The blood vessels of the supra- and infratentorial structures contained fresh red blood corpuscles. The granular layer of the cerebellar cortex was not autolytic.

Clinico-pathological features of primary brainstem death in patients with acute vascular lesions of the brainstem or cerebellum

Of 1,370 cases necropsied in our Department of Pathology during the last 10 years, there were 22 with acute infratentorial vascular lesions. Of these, four showed the clinical features of primary brainstem death lasting more than 24 hours, although it must again be stressed that the determination of apnoea was simply that spontaneous breathing had ceased,

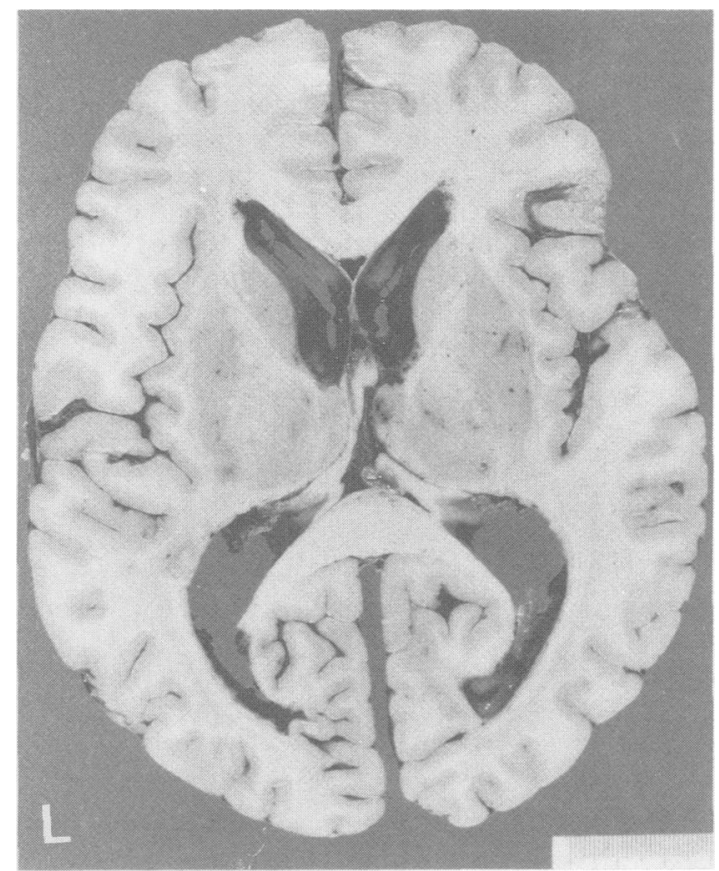

Fig 2 Transverse section of the cerebrum at the level of the splenium of corpus callosum. The lateral ventricles are slightly dilated. 


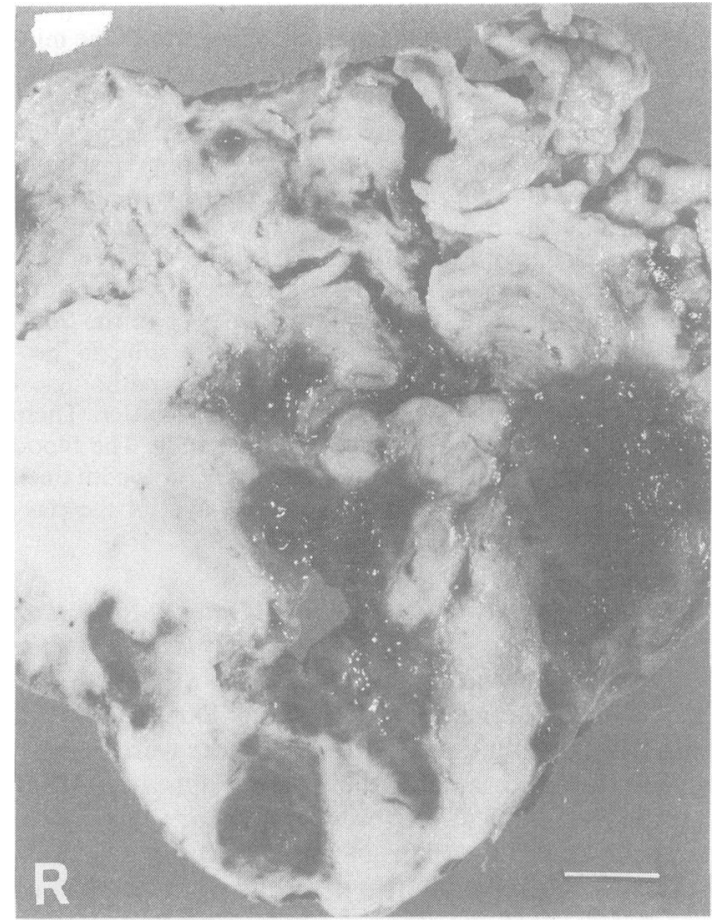

Fig 3 Transverse section of the pons and cerebellum at mid-pontine level. Extensive necrosis and haemorrhage can be seen. $B a r=1 \mathrm{~cm}$.

and that respirations were fully controlled on a ventilator. ${ }^{5}$ There were three cases with cerebellar haemorrhage and one with primary pontine haemorrhage (table). No special treatment to prolong cardiac function had been given to any of these patients. Necropsy of all four patients was performed within two hours of cardiac arrest.

Case 1 is described in the case report. The others

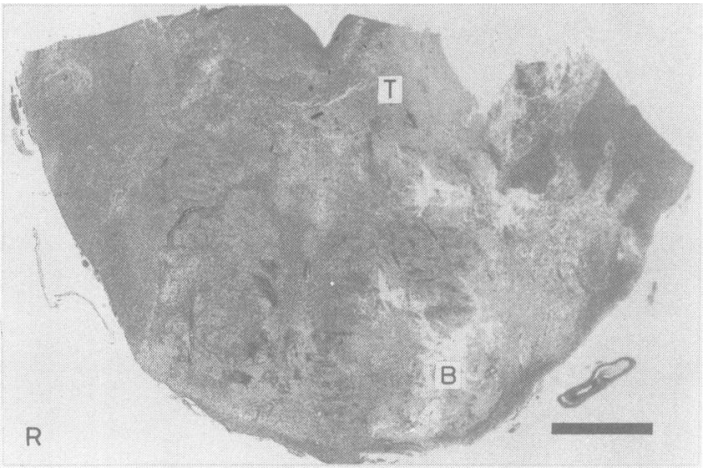

Fig 4 Transverse section of the pons at mid-pontine level. Extensive necrosis of the tegmentum $(T)$ and basis $(B)$ pontis can be seen, Haematoxylin and eosin. Bar $=5 \mathrm{~mm}$.

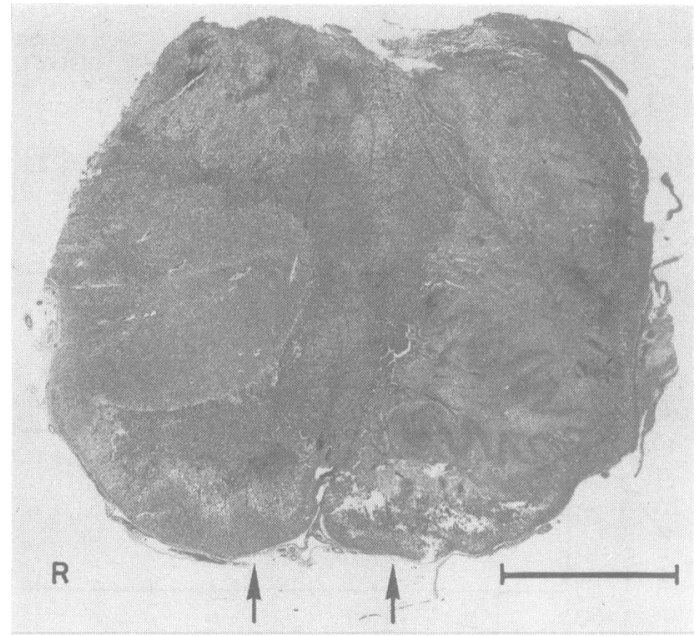

Fig 5 Transverse section of the medulla oblongata. Extensive necrosis can be seen. Arrows indicate the pyramids. Haematoxylin and eosin. Bar $=5 \mathrm{~mm}$.

were not treated surgically. Two cases with cerebellar haemorrhage (cases 2 and 3 ) and case 4 with primary pontine haemorrhage showed moderate obstructive hydrocephalus secondary to the infratentorial lesions. Case 2 suffered severe dehydration, the serum sodium reaching $169 \mathrm{mmol} / \mathrm{l}$. The brain, and especially theo infratentorial structures, were macerated. The granu lar layer of the cerebellar cortex at the periphery of the folia was autolysed. Cases 3 and 4 were not shown to be dehydrated, the serum sodium reaching 147 and $136 \mathrm{mmol} / \mathrm{l}$ respectively. The brain was not macerated in either case, and the granular layer of the cerebellar cortex was not autolytic. Three patients (cases 1, 3 and 4) whose EEG was obtained showed nonreactive alpha or slower activities during the period of primary brainstem death. The EEG had become isoelectric in case 2 one hour, and in case 425 minutes before the final cardiac arrest.

\section{Discussion}

The patients reported above showed an absence of brainstem function due to irremediable structural damage to the brainstem. They were not hypothermic or under the influence of drugs, and apart from the testing for apnoea would have satisfied the criteria of brain death laid down by the Conference of Medical Royal Colleges. ${ }^{6}$ Pathologically, the infratentorial structures especially the brainstem were seriously damaged while the supratentorial structures were fairly well preserved. In this setting, we suggest that it would be appropriate to refer to these patients as suffering from "primary brainstem death".

The severe damage restricted to the infratentorial 
Table 1 Clinical, electroencephalographic and pathological findings in four patients with vascular lesions of the brainstem or cerebellum causing primary braintem death lasting over 24 hours

\begin{tabular}{|c|c|c|c|c|c|}
\hline $\begin{array}{l}\text { Case } \\
\text { No }\end{array}$ & $\begin{array}{l}\text { Age (Years) } \\
\text { and sex }\end{array}$ & Lesions & $\begin{array}{l}\text { Duration of } \\
\text { brainstem death }\end{array}$ & $\begin{array}{l}\text { EEG during } \\
\text { brainstem death }\end{array}$ & Hydrocephalus \\
\hline 1 & 47 male & $\begin{array}{l}\text { Cerebellar haemorrhage } \\
\text { (surgically treated) }\end{array}$ & 27 days & $\begin{array}{l}\text { Theta activity } 19,7 \text { and } \\
2 \text { days before cardiac } \\
\text { arrest }\end{array}$ & $\begin{array}{l}\text { Slight (ventricular } \\
\text { drainage performed) }\end{array}$ \\
\hline 2 & 68 female & Cerebellar haemorrhage & 4 days 14 hours & $\begin{array}{l}\text { Flat } 1 \text { hour before } \\
\text { cardiac arrest }\end{array}$ & Moderate \\
\hline 3 & 72 male & Cerebellar haemorrhage & 1 day 2 hours & $\begin{array}{l}\text { Alpha activity } 18 \text { hours } \\
\text { before cardiac arrest }\end{array}$ & Moderate \\
\hline 4 & 66 male & $\begin{array}{l}\text { Primary pontine } \\
\text { haemorrhage }\end{array}$ & 1 day 4 hours & $\begin{array}{l}\text { Alpha activity } 8 \text { hours, } \\
\text { and flat } 25 \text { minutes } \\
\text { before cardiac arrest }\end{array}$ & Moderate \\
\hline
\end{tabular}

structures in case 1 is assumed to have been caused by the combined effects of the primary lesion, of raised pressure in the infratentorial compartment (due to the haemorrhage), and of a significant fall in systemic blood pressure shortly after the onset of the stroke. The raised pressure in the infratentorial compartment was relieved by the evacuation of the haematoma and extensive craniectomy, while ventricular drainage relieved circulatory disturbance in the suptratentorial compartment caused by obstructive hydrocephalus.

The granular layer of the cerebellar cortex was not autolytic in case 1 . In classical brain death absence of blood flow during a period lasting a little more than a day is known to induce autolysis of the granular layer of the cerebellar cortex. ${ }^{5}$ The cerebrum was not macerated. The lesions of the supra- and infratentorial structures showed active tissue responses and the blood vessels contained fresh red blood corpuscles. These findings are consistent with the restoration and persistence of supra- and infratentorial blood flow during the period of clinical brainstem death. ${ }^{57}$

Of the four cases with acute vascular lesions of the brainstem or cerebellum showing signs of a nonfunctioning brainstem for more than 24 hours all showed obstructive hydrocephalus. In primary brainstem death such obstructive hydrocephalus seems in fact to be the major cause of circulatory problems in the supratentorial compartment. Without drainage of the lateral ventricles the function of the supratentorial structures is destined to fail, resulting in an isoelectric EEG and in diabetes insipidus ${ }^{8}$ (that is in a clinical picture closely resembling the much more common "whole brain death"). The fact that only a small proportion of our cases developed the latter complication is consistent with the fact that in most of them a supratentorial circulation was maintained. But even if ventricular drainage is performed raised pressure in the infratentorial compartment could damage the cerebrum by upward herniation causing supratentorial circulatory disturbances.

The persistence of EEG activity was observed during the period of primary brainstem death in three patients in whom an EEG was obtained. Although clinically brainstem death is very different from "alpha-coma" (where residual signs of brainstem function persist) our findings are consistent with the previous observation that EEG patterns containing alpha activity may persist in comatose patients with acute vascular lesions of the brainstem.

In conclusion, the present clinico-pathological study show's that structural damage with irreversible loss of all testable brainstem function may occur in patients with acute vascular lesions of the brainstem or cerebellum. A specific circumstance (namely an acute vascular lesion of the brainstem or cerebellum treated by suboccipital decompressive craniectomy and ventricular drainage of the lateral ventricles) seems to be the prerequisite for the development of primary brainstem death with prolonged somatic survival.

A recent review from $\operatorname{Japan}^{10}$ has outlined the evolution of the criteria of brain death in our country, and has stressed the difficulties still met in getting brain death accepted as death. Opinion is beginning to change, however. It seems to us that if by death one means "the irreversible loss of the capacity to be conscious combined with the irreversible loss of the capacity to breathe spontaneously (and hence to maintain a spontaneous heart beat)" ${ }^{1}$ then brainstem death is death, irrespective of whether the death of the brainstem is a repercussion of pathological processes taking place above the tentorium or is a primary infratentorial event, as described in this paper.

This work was supported by a research grant for cardiovascular disease (60-2) from the ministry of health and welfare.

\section{References}

1 Pallis C. Brainstem death: the evolution of a concept. In: Morris PJ ed. Kidney Transplantation. London: Grune and Stratton, 1984:102-27. 
2 Pallis C. From brain death to brainstem death. $\mathrm{Br} \mathrm{Med} J$ 1982;285:1487-90.

3 Ferbert A, Buchner H, Ringelstein E B, Hacke W. Isolated brainstem death. Case report with demonstration of preserved visual evoked potentials (VEPs). Electroencephalogr Clin Neurophysiol 1986;65:157-60.

4 Darby J, Yonas H, Brenner R P. Brainstem death with persistent EEG activity: Evaluation by xenonenhanced computed tomography. Crit Care Med 1987;15:519-21.

5 Ogata J, Yutani C, Imakita M, et al. Autolysis of the granular layer of the cerebellar cortex in brain death. Acta Neuropathol 1986;70:75-78.

6 Diagnosis of brian death. Statement issued by the Honorary Secretary of the Conference of Medical Royal
Colleges and their Faculties in the United Kingdom on 11 October 1976. Br Med J 1976;2:1187-8.

7 Pearson J, Korein J, Harris J H, Wichter M, Braunstein P. Brain death. II. Neuropathological correlation with the radioisotopic bolus technique for evaluation of critical deficit of cerebral blood flow. Ann Neurol 1977;2:206-10.

8 Pallis C. Diabetes insipidus with brain death. Neurology 1985;35:1086-7.

9 Chase T N, Moretti L, Prensky A. L. Clinical and electroencephalographic manifestations of vascular lesions of the pons. Neurology 1968;18:357-68.

10 Takeuchi K, Takeshita H, Takakura K, et al. Evolution of criteria for determination of brain death in Japan. Acta Neurochir 1987;87:93-98. 